\title{
Regularities of visible photoluminescence creation in low-dimensional silicon structures
}

\author{
E.G. Manoilov \\ V. Lashkaryov Institute of Semiconductor Physics, NAS of Ukraine, 45 prospect Nauky, 03028 Kyiv, Ukraine \\ Phone: +38(044) 265 6230; e-mail: dept_5@isp.kiev.ua
}

\begin{abstract}
The evolution of time-resolved photoluminescence (PL) spectra in Au-doped nanocrystalline silicon films produced by laser ablation has been studied. The PL spectra with a relaxation time of nanoseconds are broad; they lie in the energy range 1.4-3.2 eV with a peak at 2.4-2.8 eV. At the longest times of tens of microseconds, the spectra become narrower, with a peak at $1.6 \mathrm{eV}$. At intermediate times, two bands are observed: low-energy $(1.6 \mathrm{eV})$ and high-energy, with the peak shifting from 2.7 to $2.1 \mathrm{eV}$ with time increasing. The data are discussed in terms of quantum confinement, dielectric amplification, and manifestation of kinetically coupled electron-hole and exciton subsystems. Ions and atoms of gold passivate dangling bonds at Si surface and serve as catalysts for oxidation of nanocrystals.
\end{abstract}

Keywords: nanocrystalline silicon, time-resolved photoluminescence, quantum confinement, dielectric amplification, metal doping.

Paper received 27.05.03; accepted for publication 17.06.03.

\section{Introduction}

Silicon nanocrystals (NC) dispersed in a dielectric medium, typically in silicon oxide $\mathrm{SiO}_{2}(0<x \leq 2)$, attract much interest owing to their efficient photoluminescence (PL) in the visible spectral range at room temperature and the possibility of observing quantum-confinement effects. Porous silicon (por-Si) and nanocrystalline silicon (nc-Si) films, exhibiting PL in the visible spectral range, belong to low-dimension structures. The majority of studies of PL in nc-Si have been concerned with static spectra of PL from por-Si produced by anodizing, and a far smaller number, with spectra of time-resolved PL (TRPL) from nc-Si films produced by glow discharge decomposition, sputtering, ion implantation, spark discharge, laser pyrolysis of silane, laser ablation, etc. PL spectra are, as a rule, complex, covering wide ranges on the energy $(h v=1.4-3.2 \mathrm{eV})$ and time scales $\left(\tau=10^{-12}\right.$ $10^{-3} \mathrm{~s}$ ) [1 - 4]. The PL spectra of Si nanocrystals, in which the fast nonradiative recombination channel associated with neutral dangling bonds on NC surface is suppressed, are more informative with respect to recombination mechanisms. In por-Si produced by chemical etching, the formation conditions ensure passivation of dangling bonds by hydrogen, oxygen, and hydroxy ions. Special efforts in the passivation of dangling bonds should be made to reveal the features concealed in nc-Si PL spectra by the surface nonradiative recombination channel.

In the present paper study, the TRPL spectra of these nc-Si films are analyzed, including their evolution with the PL relaxation time and their dependence on the size of Si nanocrystals. Our aim is to investigate the recombination mechanisms responsible for the PL spectra of ncSi films fabricated by pulsed laser ablation (PLA). Fathoming these physical regularities will unclose ways to solve technological problems of magnifying the intensity and stability of PL low-scale Si structures.

\section{Experimental}

Photoluminescent films were obtained by PLA in vacuum. The insert in Fig. 1 schematically shows the vacuum chamber. The beam 1 of a Q-switched $\mathrm{Nd}^{3+}$ :YAG laser, emitting at $1.06 \mu \mathrm{m}$ with a $10 \mathrm{~ns}$ pulse width, $20 \mathrm{~J} / \mathrm{cm}^{2}$ energy density, and $25 \mathrm{~Hz}$ repetition rate, scanned the target 5 in the form of a single-crystal $\mathrm{Si}$ (c-Si) wafer. The deposition was carried out in the atmosphere of an inert (He, Ar) or reactive $\left(\mathrm{O}_{2}\right)$ gas under a pressure of $10^{-1}-20 \mathrm{~Pa}$. 


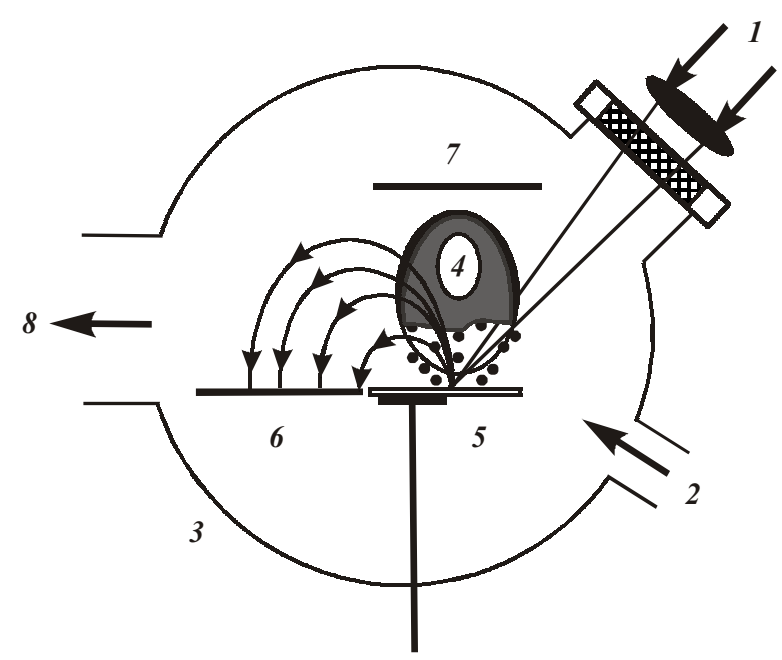

Fig. 1. Schematic of the PLA method: $l$ is the laser beam; $2-$ inlet of the inert gas; 3 - vacuum chamber; 4 - erosion plume; 5 target; 6 and 7 - substrates; 8 - vacuum pump.

Films of the first type were deposited from the direct flux of particles from the erosion plume 4 onto the substrate 7 placed opposite to the target in parallel to its plane [5]. Films of the second type were deposited from the reverse flux of the torch particles onto the substrate 6 lying in the plane of the target [6]. In the latter case, selection of Si nanocrystals by their size occurred, with coarser NC formed closer to the axis of the erosion torch. For doping nc-Si films with gold, a thin $(\sim 80 \mathrm{~nm})$ gold layer was deposited onto the c-Si target. The film growth rate was $10-20 \mathrm{~nm} / \mathrm{min}$. During deposition onto a substrate placed in the plane of the target, the film thickness decreased from $\sim 1500$ down to $\sim 300 \mathrm{~nm}$ with the distance from the erosion torch axis increasing up to $10-12 \mathrm{~mm}$. The substrates were c-Si, silica ceramics, quartz, mica, etc.

\section{Results and discussion}

The PL spectra are broad, lie in the photon energy range $h v=1.4-3.2 \mathrm{eV}$, and exhibit wide peaks at $2.4-2.6 \mathrm{eV}$ with shoulders at low (1.8-2.2 eV) and high energies (2.8$3.0 \mathrm{eV})$. The PL relaxation time $\tau$ does not exceed $50 \mathrm{ns,}$ which indicates that the radiative recombination is strongly overlapped with the nonradiative process. With NC size decreasing, the PL intensity $I_{P L}$ increases, and the spectral peak shifts from 2.45 to $2.55 \mathrm{eV}$ (Fig. 2), which is indicative of the quantum confinement effect. Spectral broadening is attributed to scatter of the sizes and shapes of nanocrystals and the diversity of dielectric properties in their environment.

Since the quantum efficiency of films was not higher than several percent and correlation was observed between the intensity $I_{P L}$ as well as PL relaxation time $\tau$ at temperatures $T>150 \mathrm{~K}$ [7], $\tau$ is defined by the nonradiative recombination time $\left(\tau_{n R}\right)$.

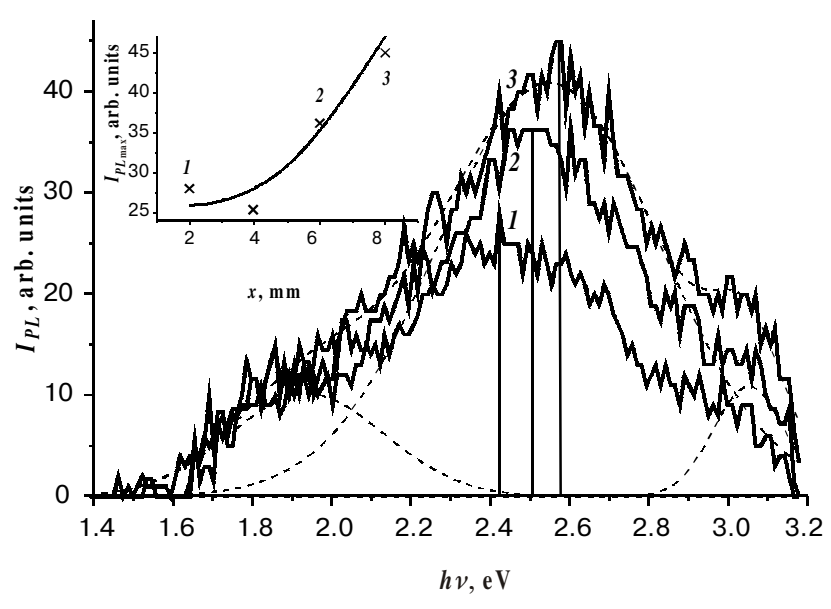

Fig. 2. TRPL spectra for freshly fabricated samples with NC size $d_{1}>d_{2}>d_{3}$.

Slow component in PL spectrum of nc-Si appearing as a result of oxidizing it in air or at heat treatment in vacuum at $10-3 \mathrm{~Pa}$ and attained $1.5-2 \mu \mathrm{s}$. Thus the magnification of PL intensity that testified to partial inhibition of the channel of nonradiative recombination was observed, too.

Thus, for transpiring that hides in PL spectra of nc-Si films a nonradiative surface channel of recombination, it was necessary to learn effectively and directly to passivate neutral dangling bonds.

The opportunity of magnification of the time of nonradiative recombination, intensity, PL stability, and also decrease of density of charge carrier traps in nc-Si the films obtained by PLA has been shown, by a passivation of $\mathrm{Si}$ dangling bonds by atoms of electropositive metals during formation of films. The greatest effect has been obtained when doping nc-Si films by gold. The increase in $\tau_{n R}$ upon passivation of NCs with gold is due to the high electron affinity $(2.3 \mathrm{eV})$ and ionization energy $(9.26 \mathrm{eV})$ of $\mathrm{Au}$ atoms (Fig. 3). Therefore, not only positive ions, but also gold atoms effectively capture electrons from dangling bonds at the surface of Si nanocrystals, which are passivated in such manner.

The temporal evolution of TRPL spectra, revealed owing to saturation of dangling with gold, is shown in Fig. 4, and 5. It can be seen that, at the relaxation time of $250 \mathrm{~ns}$, the spectral peaks fall in the high-energy (2.0$2.8 \mathrm{eV}$ ) range, whereas with time increasing they shift to a lower energy (2.0-2.5 eV). Two bands are observed, at low $(1.6 \mathrm{eV})$ and high $(2.0-2.4 \mathrm{eV})$ energies. The intensity of the low-energy band is higher at maximum relaxation times of several tens of microseconds, and the highenergy band dominates at shorter times.

Such temporal behavior of the spectra can be attributed to the manifestation in radiative recombination of two kinetically coupled subsystems: electron-hole pairs and excitons, with quantum confinement and dielectric amplification effects observed [1-4,7,8]. The decreasing of the NC size results in an increase in the oscillator strength and in a decrease in the relaxation time of 


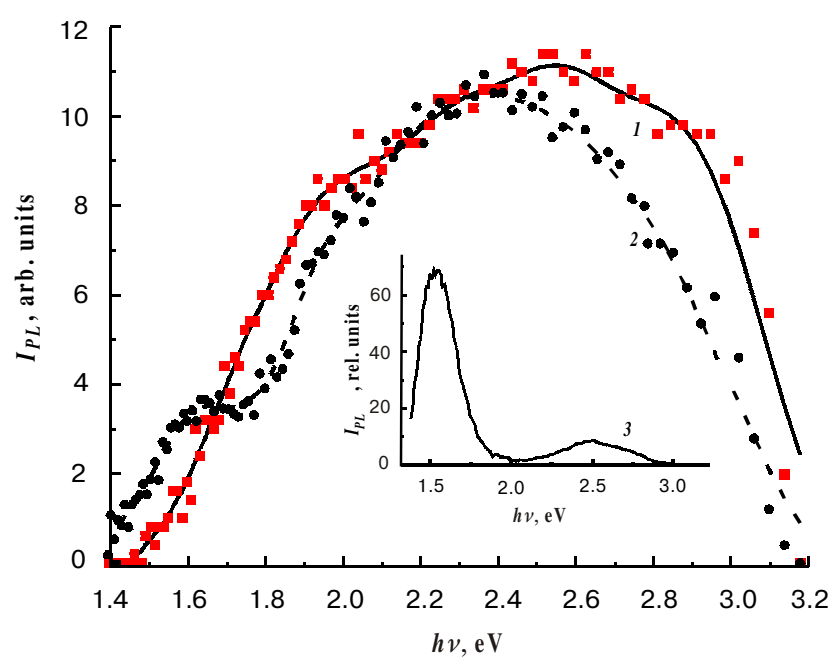

Fig. 3. TRPL spectra for $(I)$ undoped and $(2,3)$ Au-doped nc-Si films deposited from the direct flux of the torch particles. PL relaxation time $\tau$. $1-<50 \mathrm{~ns}, 2-<250 \mathrm{~ns}$, and $3-250 \mathrm{~ns}<\tau<10 \mu \mathrm{s}$.

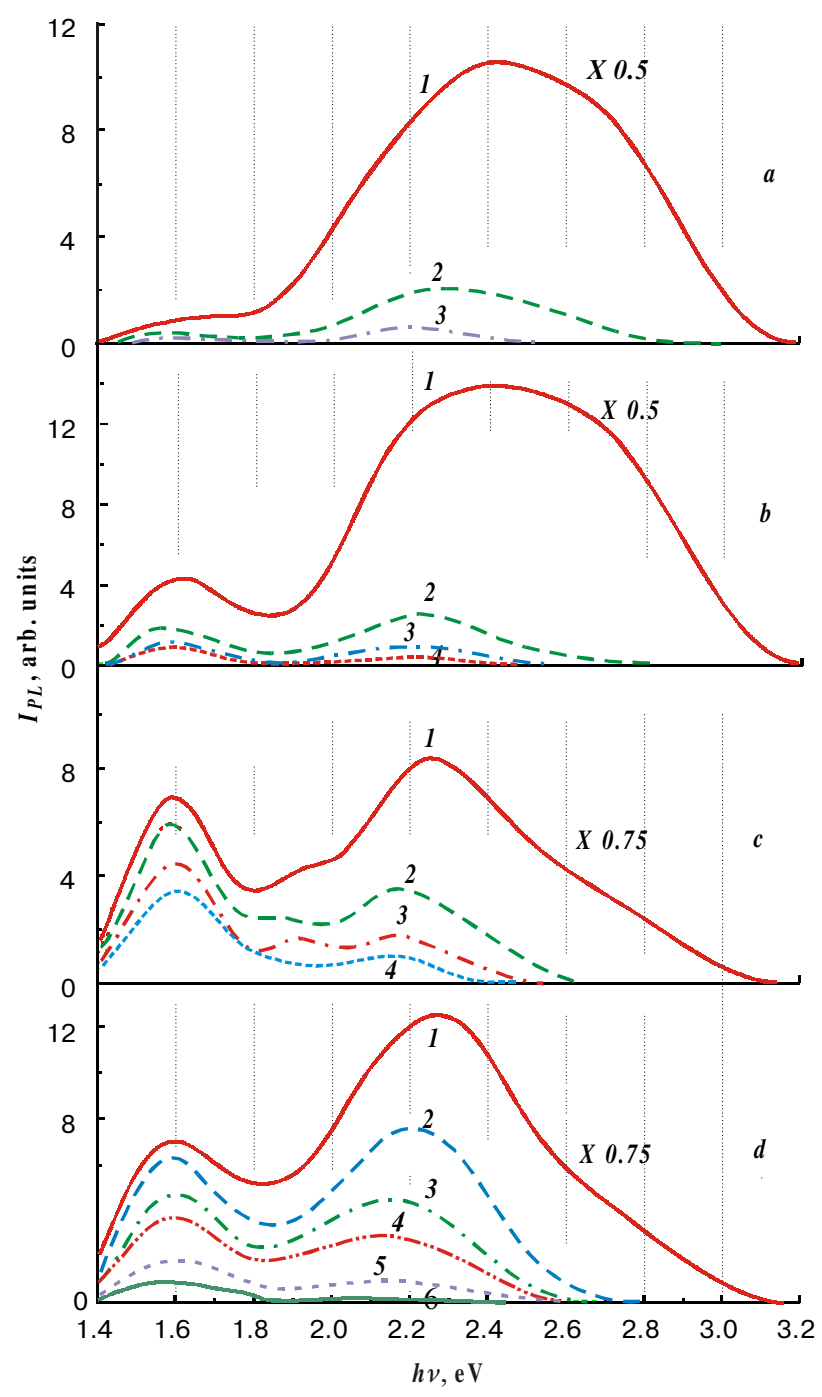

Fig. 4. TRPL spectra from four points $(a-d)$ of Au-doped nc-Si films. PL relaxation time: $1-250 \mathrm{~ns}<\tau<500 \mathrm{~ns}, 2-500 \mathrm{~ns}<\tau<750 \mathrm{~ns}$, $3-750 \mathrm{~ns}<\tau<1 \mu \mathrm{s}, 4-1 \mu \mathrm{s}<\tau<1.25 \mu \mathrm{s}, 5-1.25 \mu \mathrm{s}<\tau<1.5 \mu \mathrm{s}$, and $6-1.5 \mu \mathrm{s}<\tau<1.75 \mu \mathrm{s}$.

SQO, 6(3), 2003

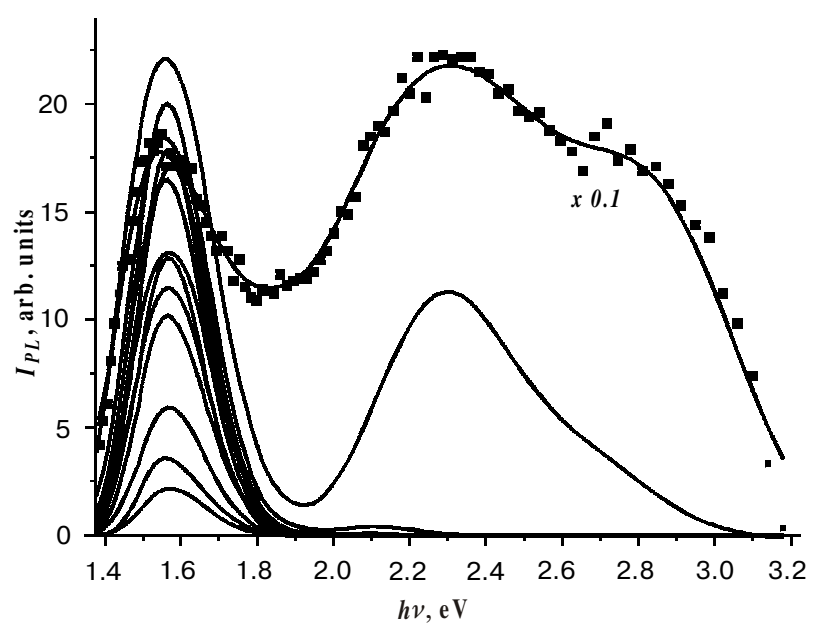

Fig. 5. TRPL spectra integrated over the film area for an Audoped nc-Si film deposited from the reverse flux of particles and additionally oxidized by keeping in air for several months. Spectra recorded with times of the PL relaxation increasing: $1-$ $\tau<250 \mathrm{~ns}$; the lowest curve, $10.5 \mu \mathrm{s}<\tau<12 \mu \mathrm{s}$.

radiative recombination for nonequilibrium carriers on the quantum-confinement levels of Si nanocrystals. Due to dielectric amplification, the exciton binding energy can become as high as $1 \mathrm{eV}$, with the excitonic emission defined by its singlet and triplet states lying in the lowenergy range and its intensity increasing [8]. This allows us to attribute the high-energy (1.9-3.2 eV) band mainly to the electron-hole pair emission and the low-energy band $(1.4-1.8 \mathrm{eV})$ to excitonic emission.

The enhancement of exciton recombination in Audoped films occurs due to the fact that Au ions and atoms capture not only electrons from dangling bonds, but, in further adsorption, also the valence electrons of weakened $\mathrm{Si}-\mathrm{Si}$ bonds at the NC surface. By rupturing these bonds, gold contributes to the oxidation of NCs [9]. The catalytic activation by gold of the oxidation process on the surface of $\mathrm{Si} / \mathrm{SiO}_{\mathrm{x}} \mathrm{NCs}$ favors the formation of dioxide $\mathrm{SiO}_{2}$ envelopes of $\mathrm{NCs}$, instead of $\mathrm{SiO}_{\mathrm{x}}(0<\mathrm{x}<2)$. This raises the potential barrier and reduces the dielectric constant of the envelope and the NC size. All of these factors favor the quantum-confinement and dielectricamplification effects, and, consequently, lead to an increase in the excitonic component in PL. In this case, the role of Au consists in that it enhances the effect of oxygen, which is observed in oxidized por-Si layers characterized by a higher PL intensity and longer relaxation time.

Data characterizing the PL stability are shown in Fig. 6 that represents the variation in PL intensity with time. Under continuous laser irradiation of undoped films, PL intensity decreases by $40-45 \%$ in the first $20 \mathrm{~min}$ (curve 1). In contrast, the films doped with Au exhibit much higher stability, with PL intensity decreasing by no more than $5 \%$ (curve 2). The Au-doped films are also more stable than undoped ones when exposed to air for a long 
E.G. Manoilov: Regularities of visible photoluminescence ...

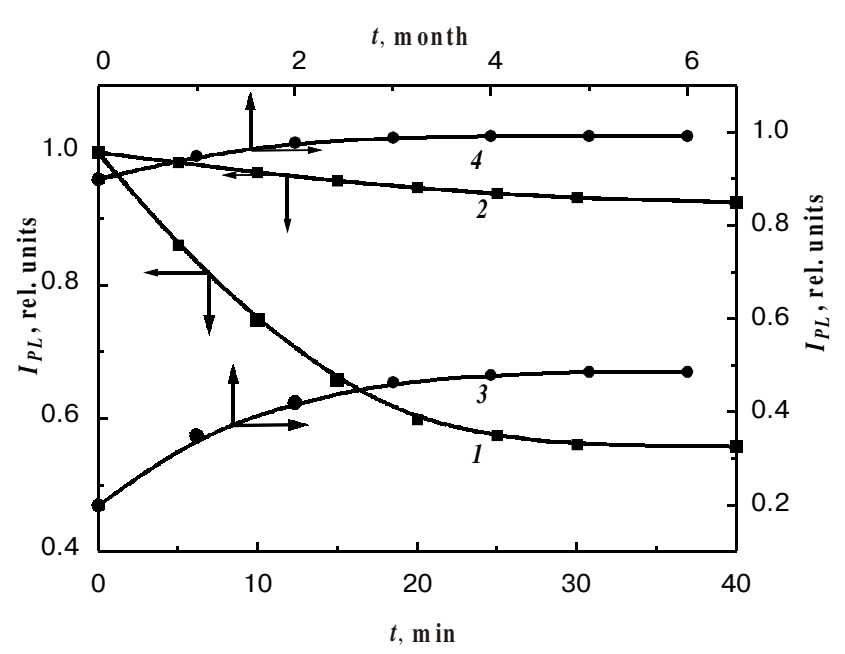

Fig. 6. Variation of the PL intensity with time $(1,2)$ under irradiation of the samples by pulses from an $N_{2}$ laser and $(3,4)$ upon exposure to air; curves $I$ and 3 correspond to undoped nc-Si films, curves 2 and 4 correspond to Au-doped ones.

period of time (curves 3, 4). For instance, one can see from Fig. 2 that PL intensity of undoped films increases by $\sim 30 \%$ after being kept in air for half a year; this takes place due to the gradual process of further oxidation of $\mathrm{SiO}_{2}$ layers. Meanwhile, the PL intensity of $\mathrm{Au}$-doped films varies with time by $5-10 \%$ at most, since in this case there is substantial initial coverage of Si nanocrystals with $\mathrm{SiO}_{2}$.

\section{Conclusions}

The possibility of observing the evolution of PL spectra at relaxation times up to tens of microseconds in nanocrystalline silicon films deposited by pulsed laser ablation and doped with gold has been demonstrated. At short times, broad spectra in the energy range $h v=1.4-3.2 \mathrm{eV}$ are ob- served. With increasing relaxation time, two spectral bands appear with peaks at 1.6 and $2.1-2.5 \mathrm{eV}$. The high-energy band dominates at short times, and only the lowenergy band peaked at $1.6 \mathrm{eV}$ is seen at the longest relaxation times. The results are discussed in terms of the quantum-confinement and dielectric-amplification effects and in terms of the involvement of kinetically coupled electron-hole (band at 1.8-3.2 eV) and exciton (band at $1.6 \mathrm{eV}$ ) subsystems in the recombination process. The detected physical regularity of the luminescent processes in nc-Si films have allowed to work out a series of technological receptions to increase intensity and PL stability.

\section{References}

1. A.G. Cullis, L.T. Canham, P.D.J. Calcott, The structural and luminescence properties of porous silicon // Appl. Phys. Rev., 82(3), pp. 909-965 (1997).

2. D. Kovalev, H. Mekler, G. Polisski, F. Koch, Optical properties of Si nanocrystals // Phys. Stat. Sol. B., 215, pp. 871932 (1999).

3. Y. Kanemitsu, Light emission from porous silicon and related materials // Phys. Rep., 263, pp. 1-91 (1995).

4. O. Bisi, S. Ossicini, L. Pavesi, Porous silicon: a quantum sponge structure for silicon based optoelectronics // Surf. Sci. Rep., 38, pp. 1-126 (2000).

5. E.B. Kaganovich, A.A. Kudryavtsev, E.G. Manoilov, S.V. Svechnikov, I.Z. Indutnyi, Optical properties of Si nanocomposite films prepared by laser ablation // Thin Sol. Films., 349, pp. 298-302 (1999).

6. L. Patrone, D. Nelson, V.I. Safarov, M. Sentis, W. Marine, S. Giorgio, Photoluminescence of silicon nanoclusters with reduced size dispersion produced by laser ablation // J. Appl. Phys., 87(8), pp. 3829-3837 (2000).

7. A.V. Sachenko, E.B. Kaganovich, E.G. Manoilov, S.V. Svechnikov, Kinetics of exciton photoluminescence in low-dimensional silicon structures (in Russian) // Fiz. Tekhn. Poluprov., 35(12), pp. 1445-1451 (2001).

8. P.K. Kashkarov, B.V. Kamenev, E.A. Konstantinova, Dynamics of nonequilibrium charge carriers in silicon wires (in Russian) // Usp. Fiz. Nauk, 168(5), pp. 577-582 (1998).

9. V.E. Primachenko, O.V. Snitko, Physics of semiconductor surface doped with metals (in Russian), Naukova Dumka, Kiev (1988). 